\title{
Optical activation of Si nanowires using Er-doped sol-gel derived silica
}

Kiseok Suh ${ }^{1}$, Oun-Ho Park ${ }^{2}$, Byeong-Soo Bae ${ }^{2}$, Jung-Chul Lee ${ }^{3}$, Heon-Jin Choi ${ }^{4}$, Jung H. Shin ${ }^{1}$

${ }^{1}$ Department of Physics, Korea Advanced Institute of Science and Technology (KAIST), 373-1 Guseong-dong, Yuseong-gu, Daejeon, Korea

${ }^{2}$ Department of Materials Science and Engineering, KAIST, 373-1 Guseong-dong, Yuseong-gu, Daejeon, Korea

${ }^{3}$ Materials science and Technology Division, Korea Institute of Science and Technology (KIST), P.O. Box 131, Cheongryang, Seoul 130-650, Korea

${ }^{4}$ Department of Ceramics, Yonsei University, Seoul 120-749, Korea

\section{ABSTRACT}

Optical activation of $\mathrm{Si}$ nanowires (Si-NWs) using sol-gel derived Er-doped silica is investigated. Si-NWs of about $100 \mathrm{~nm}$ diameter were grown on Si substrates by the vapor-liquidsolid method using $\mathrm{Au}$ catalysts and $\mathrm{H}_{2}$ diluted $\mathrm{SiCl}_{4}$. Afterwards, Er-doped silica sol-gel solution was spin-coated, and annealed at $950{ }^{\circ} \mathrm{C}$ in flowing $\mathrm{N}_{2} / \mathrm{O}_{2}$ environment. Such Er-doped silica/Si-NWs nanocomposite is found to combine the advantages of crystalline $\mathrm{Si}$ and silica to simultaneously achieve both high carrier-mediated excitation efficiency and high $\mathrm{Er}^{3+}$ luminescence efficiency while at the same time providing high areal density $\mathrm{of}^{\mathrm{Er}^{3+}}$ and easy current injection, indicating the possibility of developing sol-gel activated Si-NWs as a new material platform for Si-based photonics.

\section{INTRODUCTION}

Efficient Si-based light emitting material has been attracting much interest because of its potential application in photonics with Si technology[1]. In particular, Er-doping of Si material has great importance due to their $\mathrm{Er}^{3+}$ intra-4f emission at $1.54 \mu \mathrm{m}$, the standard wavelength for telecommunications. After the initial report[2], Er-doped Si light emitting diodes (LEDs) operating at room temperature were demonstrated with good electrical properties. However, the luminescence efficiencies were quite poor due to their intrinsic Auger- and temperature quenching of $\mathrm{Er}^{3+}$ luminescence[3]. On the other hand, excellent optical properties were obtained by using silicon-rich-silicon-oxide (SRSO), which consists of nanocluster Si (nc-Si) embedded 
inside an $\mathrm{SiO}_{2}$ matrix[4]. Although optical gain[5] as well as efficient $\mathrm{LED}[6]$ have been demonstrated, $\mathrm{SiO}_{2}$ matrix makes current injection difficult, resulting in requirement of high voltages or thin SRSO layers for LED operation. Furthermore, excitation occurs via impact excitation by energetic carriers, which raises questions about the long-term reliability of such devices. Such problems may be solved by using Si nanowires (Si-NWs) instead, since electronic devices based on Si-NWs operating at voltages of less than 5 volts were demonstrated[7].

However, due to the limited solubility of $\mathrm{Er}$ in $\mathrm{Si}$, Er segregates at the interface during growth of Er-doped Si, and can lead to defective growth. Indeed, previous reports on Er-doping of SiNWs indicated that Er-doped Si-NWs consisted of Si core with Er-rich, defective polycrystalline surface layer with weak or no $\mathrm{Er}^{3+}$ luminescence[8]. On the other hand, there have been reports that the $\mathrm{Er}^{3+}$ can be excited by carriers over a distance of a $\mathrm{nm}$, and that the optimum location for $\mathrm{Er}^{3+}$ is not inside $\mathrm{Si}$, but in the nm-thin oxide shell right next to $\mathrm{Si}$ [9]. This suggests that rather than trying to dope Si-NWs directly, it would be preferable to coat the Si-NWs with high-quality, Er-doped silica in order to optically activate the Si-NWs. In this paper, we report on the results of such optical activation of Si-NWs using sol-gel derived Er-doped silica.

\section{EXPERIMENTAL DETAILS}

Si-NWs were grown on Si (100) wafers by VLS mechanism in a quartz tube furnace using 2 $\mathrm{nm}$ thick sputtered $\mathrm{Au}$ film as the catalyst[10]. During growth, $\mathrm{H}_{2}$ and $\mathrm{Ar}$ gases were introduced into the tube at a flow rate of $100 \mathrm{sccm}$, respectively. $\mathrm{SiCl}_{4}$ was introduced into the tube using $\mathrm{H}_{2}$ carrier gas that was bubbled through liquid $\mathrm{SiCl} 4$ held at $0{ }^{\circ} \mathrm{C}$ at a flow rate of $5 \mathrm{sccm}$. The growth temperature was $900{ }^{\circ} \mathrm{C}$. Er-doped silica sol-gel solution was prepared by first hydrolyzing tetraethylorthosilicate (TEOS, Aldrich) in a $0.05 \mathrm{M} \mathrm{HCl}$ aqueous solution with ethanol as the cosolvent. Er-doping was achieved with $\mathrm{ErCl}_{3} \cdot 6 \mathrm{H}_{2} \mathrm{O}$ (Aldrich) with a $\mathrm{ErCl}_{3} \cdot 6 \mathrm{H}_{2} \mathrm{O}$ /TEOS mol ratio of $\approx 1.0$ at. \%, corresponding to an Er concentration of 0.33 at. $\%$ in the finished film. The mixture was then stirred at RT for 1 day. The solution was spin coated on the Si-NW grown Si wafers at $1000 \mathrm{rpm}$ for $30 \mathrm{sec}$, and annealed at $450{ }^{\circ} \mathrm{C}$ for 7 hours in a flowing $\mathrm{N}_{2} / \mathrm{O}_{2}$ environment to remove volatile organic compounds. Finally, a rapid thermal anneal at $950{ }^{\circ} \mathrm{C}$ for $5 \mathrm{~min}$ in a flowing $\mathrm{N}_{2} / \mathrm{O}_{2}$ environment was used to activate erbium. The silica layer thickness was about $5 \mu \mathrm{m}$. For a comparison, sol-gel derived Er-doped pure silica film without Si-NWs was also deposited on a blank Si wafer using identical procedures. However, the silica layer thickness was only about $0.6 \mu \mathrm{m}$ in this case due the absence of Si-NWs. The $\mathrm{Er}^{3+}$ photoluminescence (PL) spectra were measured using an Ar laser, a grating monochromator, a thermo-electrically cooled InGaAs detector, and the standard lock-in technique. Low temperature 
PL spectra were measured using a closed-cycle helium cryostat, and $\mathrm{Er}^{3+} \mathrm{PL}$ decay traces were measured using a digitizing oscilloscope.

\section{DISCUSSION}

Figure 1 (a) shows a scanning-electron microscope (SEM) image of as-grown Si-NWs. We observe a dense array of straight Si-NWs of about $100 \mathrm{~nm}$ in diameter and $10 \mu \mathrm{m}$ in length. The Si-NW are not normal to the substrate, however. They lie at an angle of about 60 degrees from the surface normal. Figure 1 (b) shows an SEM image of sol-gel derived Er-doped silica film coated Si-NWs. We find that the sol-gel solution has completely penetrated the Si-NW arrays, forming a composite material of Er-doped silica interspersed with Si-NWs. Note, however, that portions of Si-NWs protrude from the silica layer, providing continuous current paths to the underlying Si substrate. Some macroscopic cracks due to shrinkage of the sol-gel derived film during drying and annealing can also be observed[11]. It should be pointed out here that the volume density of Si-NWs is estimated to be quite low, only about $2.8 \%$. However, due to the high aspect ratio of the Si-NWs and the fact that they lie at an angle, they provide about 11 times more surface area than the substrate alone.

Figure 2 shows the room temperature PL spectra of the Si-NWs and the pure Er-doped silica thin film, pumped with the 473 and $488 \mathrm{~nm}$ line of an Ar laser. The intensities are normalized by the peak intensities obtained when pumped with the $488 \mathrm{~nm}$. The $473 \mathrm{~nm}$ line was chosen because it is absorbed only by Si-NWs and not directly by $\mathrm{Er}^{3+}$ ions. $\mathrm{Er}^{3+}$ ions can absorb the photon with the wavelength of $488 \mathrm{~nm}$.

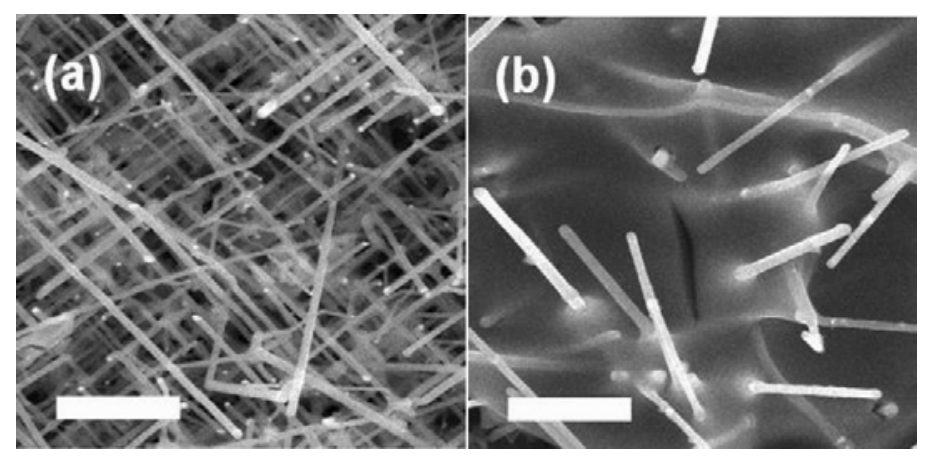

Figure 1. SEM images of (a) as-grown Si-NWs and (b) Er-doped, sol-gel-derived silica film coated on Si-NWs. Scale bar represents $2 \mu \mathrm{m}$. 


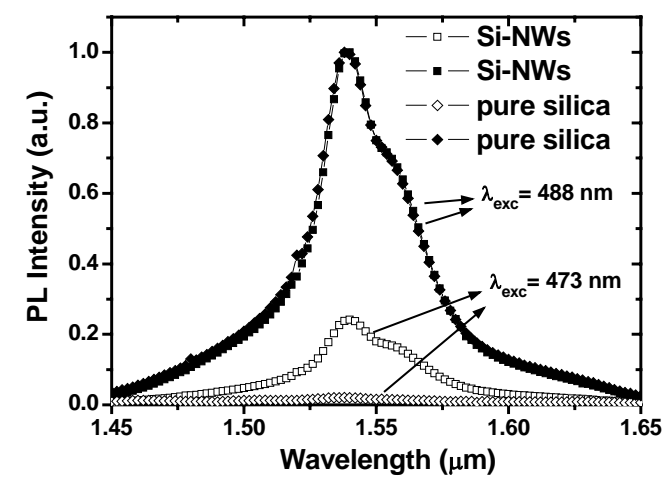

Figure 2. Room temperature PL spectra of the Si-NWs and pure Er-doped silica film

Unlike pure silica film, Si-NWs pumped with the $473 \mathrm{~nm}$ show strong $\mathrm{Er}^{3+}$ luminescence at 1.54 $\mu \mathrm{m}$, indicating energy transfer from carriers in $\mathrm{Si}-\mathrm{NWs}$ to $\mathrm{Er}^{3+}$ ions i.e., $\mathrm{Er}^{3+}$ excitation occurs via carriers only, and represents an accurate simulation of the situation under current injection.

Figure 3 shows the pump wavelength dependence of the $\mathrm{Er}^{3+}$ luminescence intensity. The intensities are normalized to the value at the pump wavelength of $515 \mathrm{~nm}$. The pure silica film without Si-NWs shows appreciable $\mathrm{Er}^{3+}$ luminescence only near the pump wavelengths of 488 and $515 \mathrm{~nm}$, reflecting the ${ }^{4} \mathrm{I}_{15 / 2} \rightarrow{ }^{4} \mathrm{~F}_{7 / 2}$ and ${ }^{4} \mathrm{I}_{15 / 2} \rightarrow{ }^{2} \mathrm{H}_{11 / 2}$ optical absorption bands of $\mathrm{Er}^{3+}$, respectively. Note that the $\mathrm{Er}^{3+}$ luminescence intensity from Si-NWs also displays strong peaks near the pump wavelengths of 488 and $515 \mathrm{~nm}$ superimposed on a near-constant background. This indicates that a large fraction of $\mathrm{Er}^{3+}$ ions in the silica film remain inaccessible to carriermediated excitation via Si-NWs, which we attribute to the low density of Si-NWs.

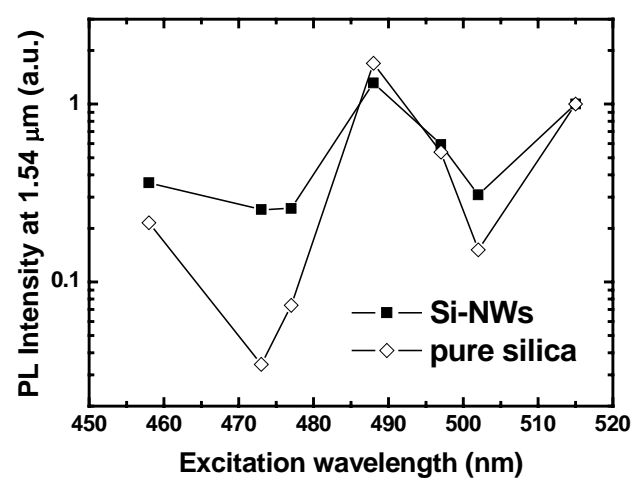

Figure 3. The pump wavelength dependence of the $\mathrm{Er}^{3+}$ luminescence intensity 
Figure 4 (a) shows the effect of temperature on the integrated $\mathrm{Er}^{3+} \mathrm{PL}$ intensities. The pure silica film was pumped using the $488 \mathrm{~nm}$ line of an Ar laser in order to directly excite the $\mathrm{Er}^{3+}$ ions. On the other hand, the Si-NWs were pumped using the $477 \mathrm{~nm}$ line of an Ar laser, which is not absorbed optically by $\mathrm{Er}^{3+}$ ions, in order to probe only those $\mathrm{Er}^{3+}$ ions that can be excited via carriers. We observe nearly identical temperature dependence of the $\mathrm{Er}^{3+} \mathrm{PL}$ intensities, decreasing by less than $10 \%$ as the temperature is raised from $25 \mathrm{~K}$ to room temperature. The temperature dependence of $\mathrm{Er}^{3+}$ luminescence lifetimes from Si-NWs and pure silica film are

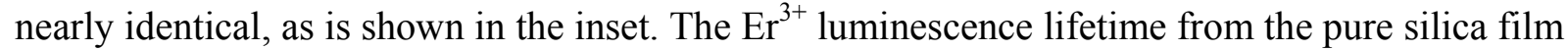
decreases from 13.6 to $11 \mathrm{msec}$ as the temperature is raised from 25 to $300 \mathrm{~K}$, while that from the Si-NWs decreases from 8.3 to $6.9 \mathrm{msec}$.

Given the large diameters of Si-NWs, such complete suppression of thermal quenching of $\mathrm{Er}^{3+}$ luminescence cannot be due to any quantum effects. In fact, PL investigations of Si-NWs did not show any quantum effects (data not shown). Yet $\mathrm{Er}^{3+}$ luminescence from bulk, crystalline Si is known to undergo severe temperature quenching[3]. Thus, we conclude that the $\mathrm{Er}^{3+}$ ions excited via carriers generated in Si-NWs are actually in the sol-gel derived Er-doped silica film that is coating the Si-NWs, in agreement with previous reports that $\mathrm{Er}^{3+}$ ions in a nm-thin silica shell around Si have both high carrier-mediated excitation efficiency and high $\mathrm{Er}^{3+}$ luminescence efficiency irrespective of quantum effects[12].
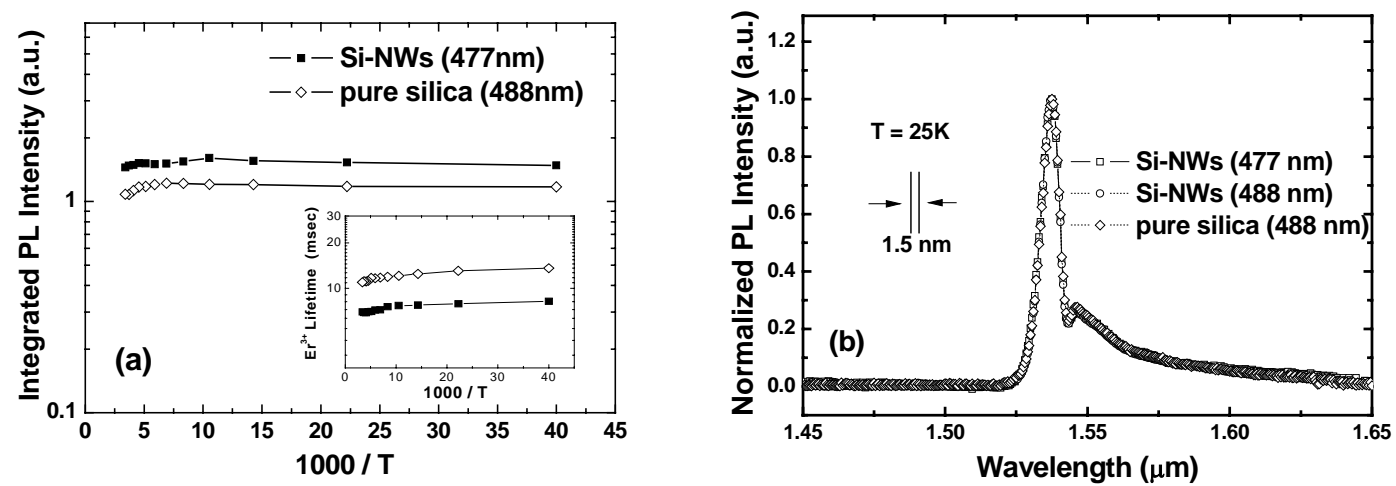

Figure 4. (a) The temperature dependence of $\mathrm{Er}^{3+}$ integrated PL intensities, showing the complete suppression of thermal quenching. The inset shows the temperature dependence of the luminescence lifetimes. (b) Normalized PL spectra of pure Er-doped silica (488 nm), with SiNWs $(488 \mathrm{~nm})$ and with Si-NWs $(477 \mathrm{~nm})$ at $25 \mathrm{~K}$. 
This conclusion is supported by the low-temperature PL spectra (Figure 4 (b)). The ${ }^{4} \mathrm{I}_{13 / 2} \rightarrow$ ${ }^{4} \mathrm{I}_{15 / 2}$ transition responsible for the $1.54 \mu \mathrm{m} \mathrm{Er}{ }^{3+}$ luminescence is a parity-forbidden transition that occurs in part due to the effects of the crystal field, and the exact shape and position of the luminescence spectra depend on the chemical and structural environment of $\operatorname{Er}[13]$. However, the $\mathrm{Er}^{3+} \mathrm{PL}$ spectra obtained from the Si-NWs and pure silica film are completely identical irrespective of the pump wavelengths, indicating that the optically-excited and carrier-excited $\mathrm{Er}^{3+}$ ions are all in the same environment - i.e., in silica.

\section{CONCLUSIONS}

We have investigated optical activation of Si nanowires by spin-coating them with a sol-gel derived Er-doped silica film. Such Er-activated Si nanowires display strong $\mathrm{Er}^{3+}$ luminescence, excited via carriers in Si nanowires efficiently, yet comparable to pure silica in luminescence efficiency, showing promise of fabricating LEDs based on Si operating at low voltages.

\section{REFERENCES}

1. See, for example, Towards the First Silicon Laser, NATO Science Series II , 93 (2003)

2. H. Ennen, J. Schneider, G. Pomrenke, and A. Axmann, Appl. Phys. Lett. 43, 943 (1983)

3. J. Palm, F. Gan, B. Zheng, J. Michel, and L. C. Kimerling, Phys. Rev. B 54, 17603 (1996)

4. J. H. Shin, M-J. Kim, S-Y. Seo, and C. Lee, Appl. Phys. Lett. 72, 1092 (1998)

5. H-S. Han, S-Y. Seo, J. H. Shin, and N. Park, Appl. Phys. Lett. 81, 3720 (2002)

6. G. Franzó, A. Irrera, E. C. Moreira, M. Miritello, F. Iacona, D. Sanfilippo, G. Di Stefano, P. G. Fallica, and F. Priolo, Appl. Phys. A 74, 1 (2002)

7. Y. Cui, and C. M. Lieber, Science 291, 851 (2001)

8. Z. Wang, and J. L. Coffer, Nano Lett. 2, 1303 (2002)

9. J-H. Jhe, J. H. Shin, K. J Kim, and D. W. Moon, Appl. Phys. Lett. 82, 4489 (2003)

10. R. S. Wagner, and W. C. Ellis, Appl. Phys. Lett. 4, 89 (1964)

11. L. H. Slooff, M. J. A. de Dood, A. van Blaaderen, and A. Polman, J. Non-crystalline Solids 296, $158(2001)$

12. T. Kimura, H. Isshiki, S. Ide, T. Shimizu, and T. Ishida, Appl. Phys. 93, 2595 (2003)

13. M. Stepikhova, W. Jantsch, G. Kocher, L. Palmetshofer, M. Shoisswohl, and H. J. Von Bardeleben, Appl. Phys. Lett. 71, 2975 (1997) 